\title{
Room-Temperature Synthesis of Hollow Polymer Microparticles with an Open Hole on the Surface and Their Application
}

\author{
Dae Hwan Kim, ${ }^{\dagger}$ Hee-Chul Woo, ${ }^{\S}$ and Mun Ho Kim ${ }^{*}{ }^{\dagger}$ \\ ${ }^{\dagger}$ Department of Polymer Engineering, Pukyong National University, 45 Yongso-ro, Nam-gu, \\ Busan 48513, Republic of Korea. E-mail: munho@pknu.ac.kr \\ ${ }^{\S}$ Department of Chemical Engineering, Pukyong National University, 45 Yongso-ro, Nam-gu, \\ Busan 48513, Republic of Korea
}

KEYWORDS. hollow particle, porous particle, polystyrene, silver nanocrystals, heterogeneous catalyst. 
Supporting Tables.

Table S1. Activity parameter, $\kappa$, values for various heterogeneous catalysts and the dependence of this parameter on the reaction rate constant and amount of catalyst used.

\begin{tabular}{|c|c|c|c|c|}
\hline Heterogeneous Catalysts & Mass [g] & $k\left[\mathrm{~s}^{-1}\right]$ & $\kappa\left[s^{-1} g^{-1}\right]$ & Ref. \\
\hline Hollow PS/Au-Ag NPs & 0.00040 & 0.0021 & 5.35 & $\mathbf{1}$ \\
\hline Hollow PS/Pt-Ag NPs & 0.00040 & 0.0046 & 11.43 & 1 \\
\hline Cross-linked PS/Au NPs & 0.10000 & 0.0250 & 0.25 & 35 \\
\hline Layer double hydroxide/Au-Ag NPs & 0.00100 & 0.0005 & 0.48 & 36 \\
\hline Spindle-shaped PANI/Au NPs & 0.03150 & 0.0404 & 1.28 & 37 \\
\hline $\mathrm{SiO}_{2} @$ polypyrrole/Au NPs & 0.03150 & 0.0442 & 1.40 & 38 \\
\hline Metal-organic framework/Au-Ag NPs & 0.00346 & 0.0049 & 1.44 & 39 \\
\hline Heterocycle-modified polystyrene/Au NPs & 0.01500 & 0.0307 & 2.05 & 40 \\
\hline PMMA/Au NPs & 0.00350 & 0.0079 & 2.26 & 41 \\
\hline Self-assembly of surface-modifided Au NPs & 0.00090 & 0.0026 & 2.86 & 42 \\
\hline CNT@PZS/Au NPs & 0.00030 & 0.0018 & 5.94 & 43 \\
\hline Carbon Nanofibers/Ag NPs & 0.00100 & 0.0062 & 6.20 & 44 \\
\hline MWCNT@S. lavandulifolia/ Ag NPs & 0.00300 & 0.0192 & 6.40 & 45 \\
\hline Carbon spheres/Au-Ag NPs & 0.00100 & 0.0065 & 6.46 & 46 \\
\hline Polyaniline nanofibers/Ag NPs & 0.00270 & 0.0214 & 7.92 & 47 \\
\hline Silica nanosheets/Ag NPs & 0.01000 & 0.0801 & 8.02 & 48 \\
\hline $\mathrm{Fe}_{3} \mathrm{O}_{4} @ \mathrm{TiO}_{2} / \mathrm{Au} \mathrm{NPs}$ & 0.00100 & 0.0103 & 10.29 & 49 \\
\hline PS with an open hole/Ag NPs & 0.00012 & 0.0017 & 14.50 & This work \\
\hline
\end{tabular}




\section{Supporting Figures}

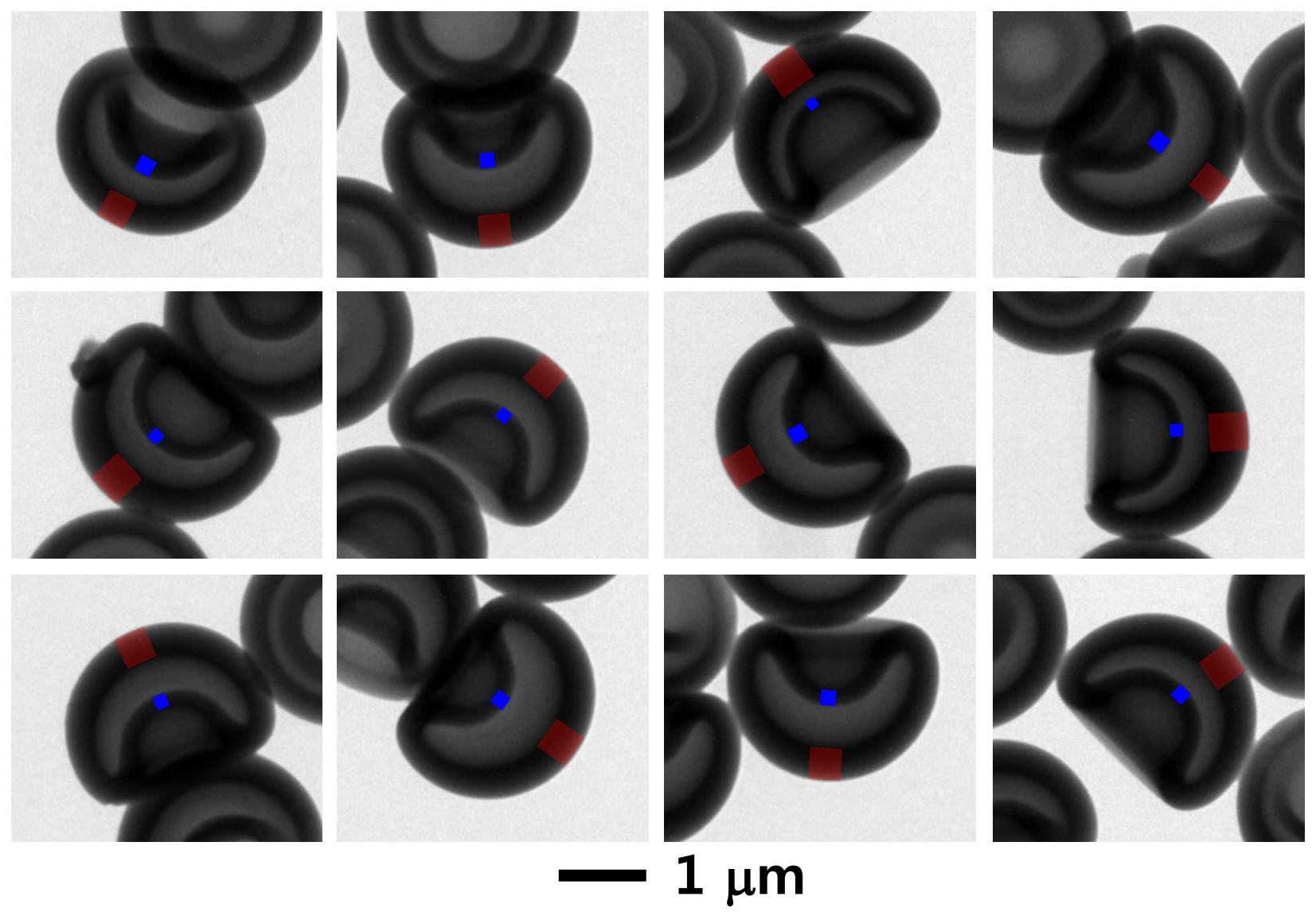

Figure S1. TEM images of PS microparticles dispersed in a water-toluene mixture for $1 \mathrm{~min}$, followed by an evaporation process at room temperature. The red and blue cubes were used to measure the average shell thicknesses in the dimple part and in the convex part of the dimple particles. 


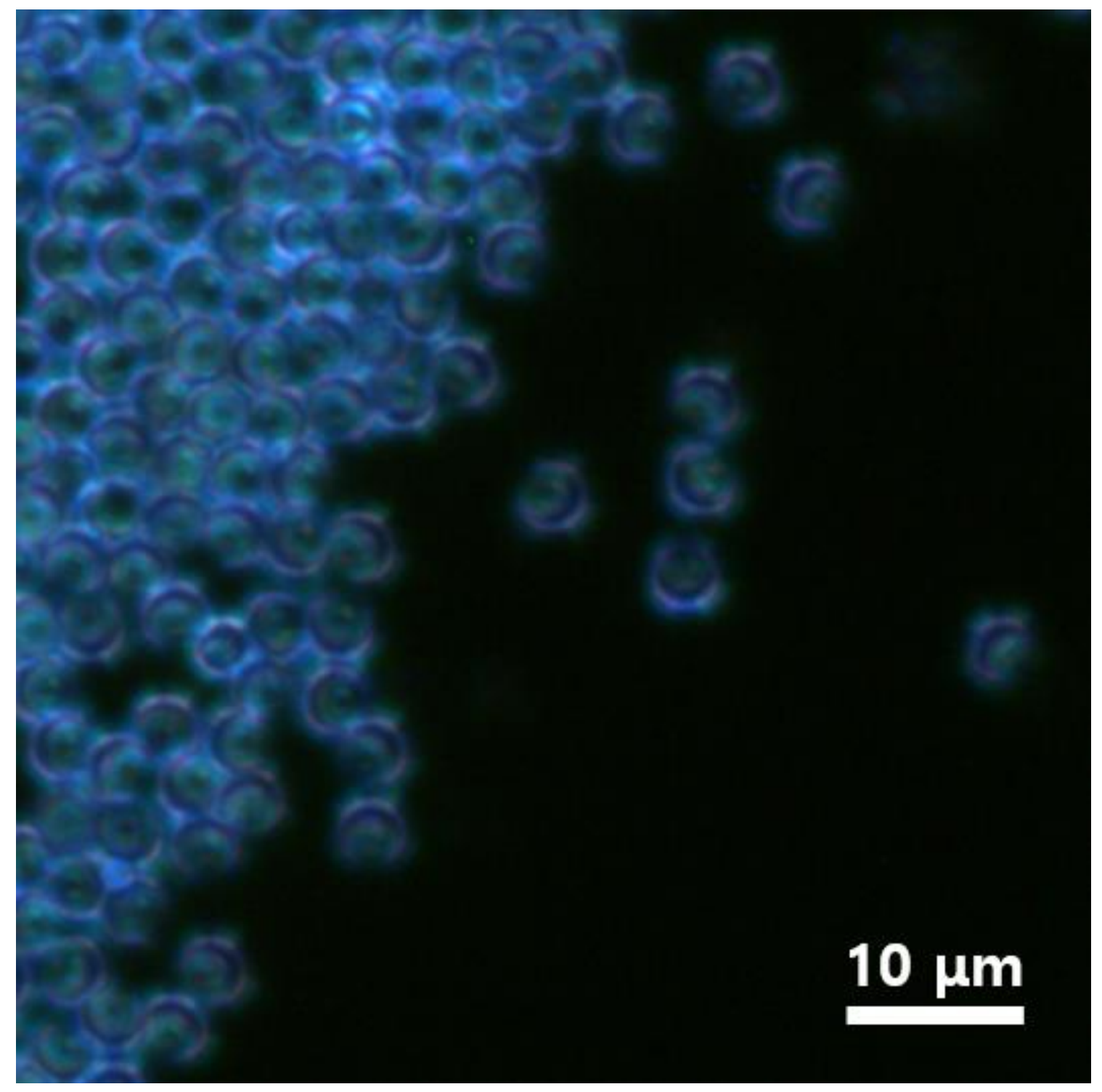

Figure S2. Optical microcopy image of PS microparticles dispersed in a water-toluene mixture for $10 \mathrm{~min}$. 

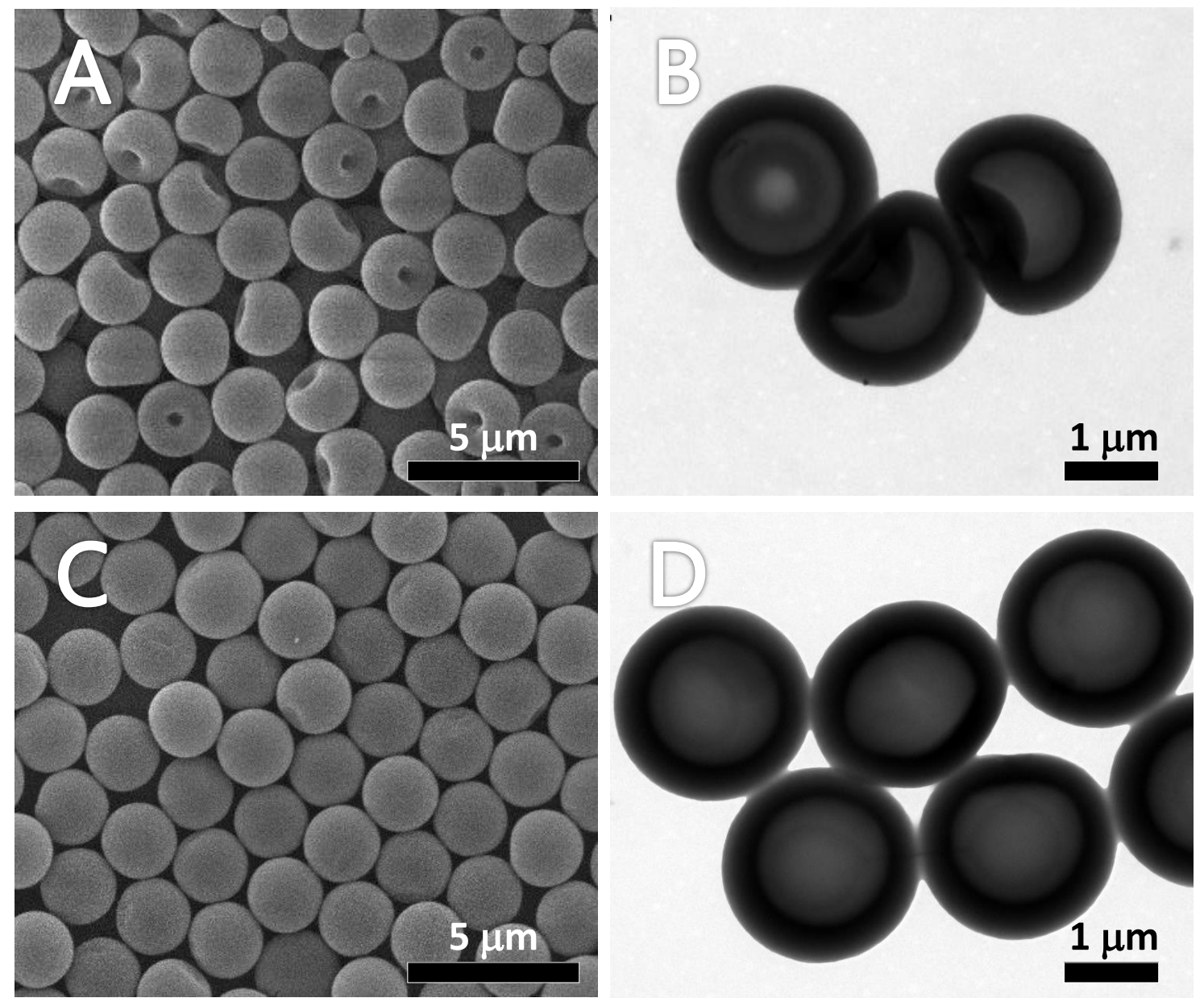

Figure S3. (A),(C) SEM and (B),(D) TEM images of PS seeds dispersed in water at (A-B) 60 ${ }^{\circ} \mathrm{C}$ and $(\mathrm{C}-\mathrm{D}) 70{ }^{\circ} \mathrm{C}$ for $12 \mathrm{~h}$. 


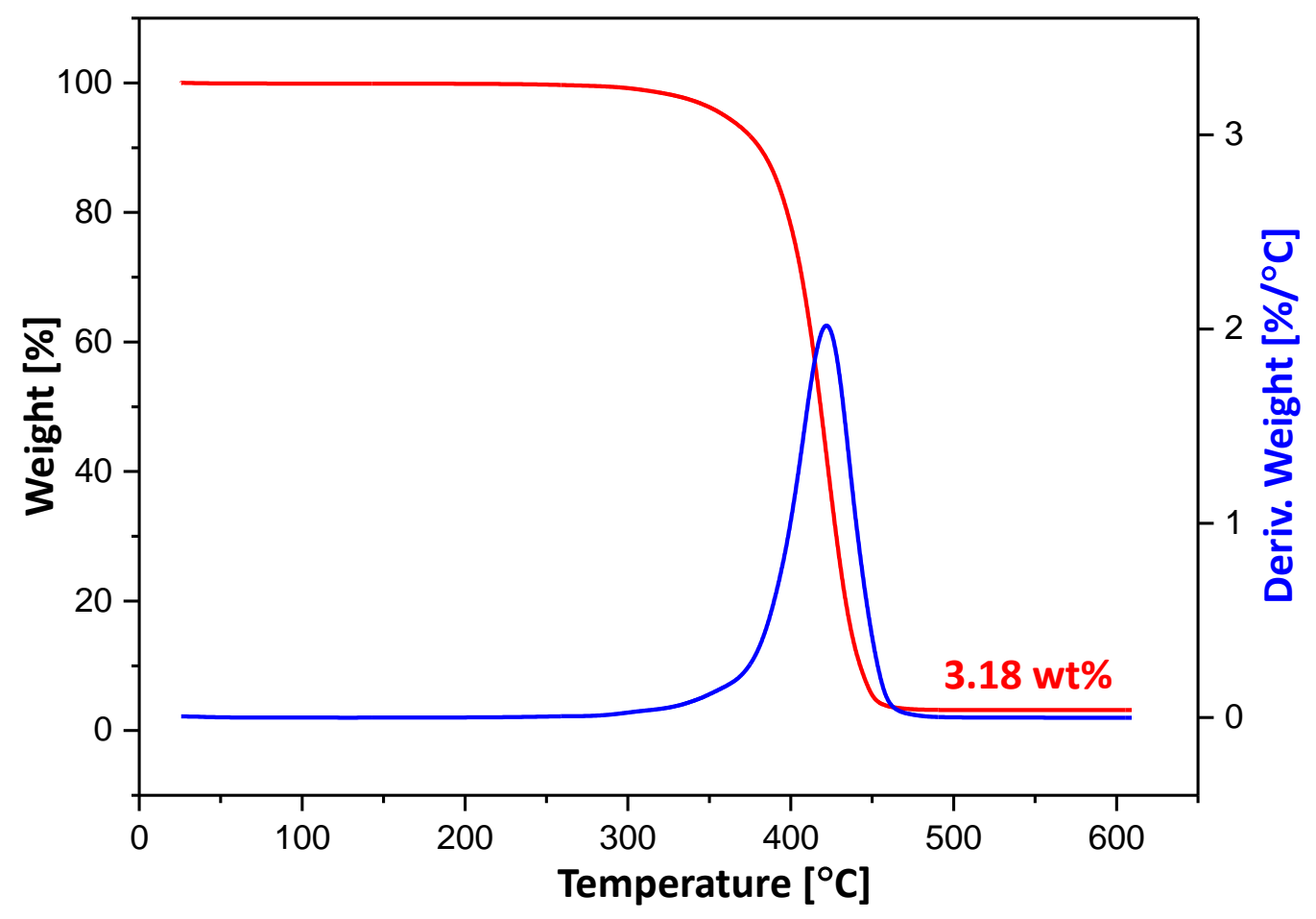

Figure S4. TGA data of Ag nanocrystals/pored PS hybrid microparticles, as obtained with the sample under nitrogen and heated at $10{ }^{\circ} \mathrm{C} / \mathrm{min}$. 


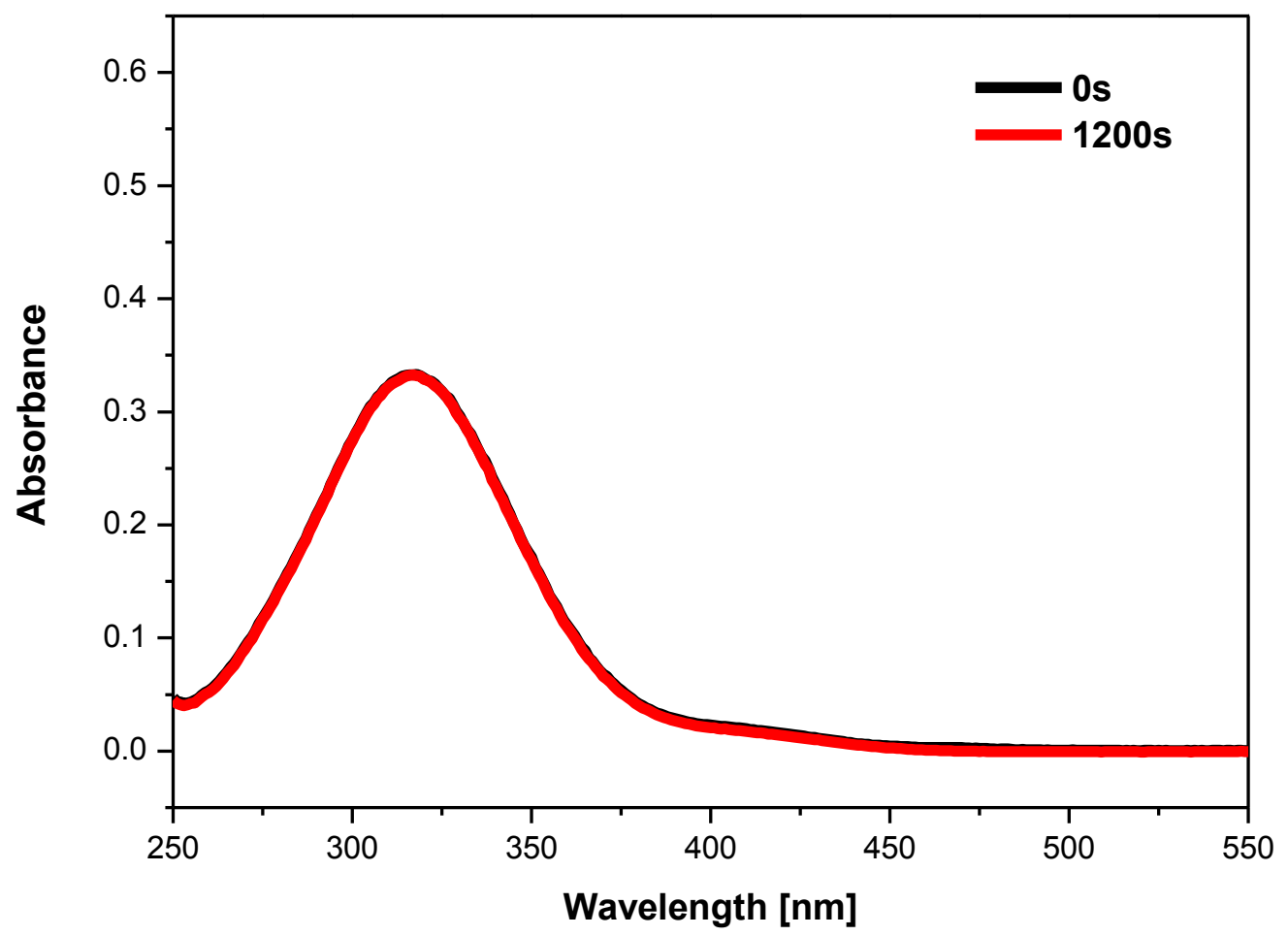

Figure S5. UV-Vis absorption spectra after Ag nanocrystals/pored hollow PS hybrid particles were added to the solution containing 4-NP (without $\mathrm{NaBH}_{4}$ ). 


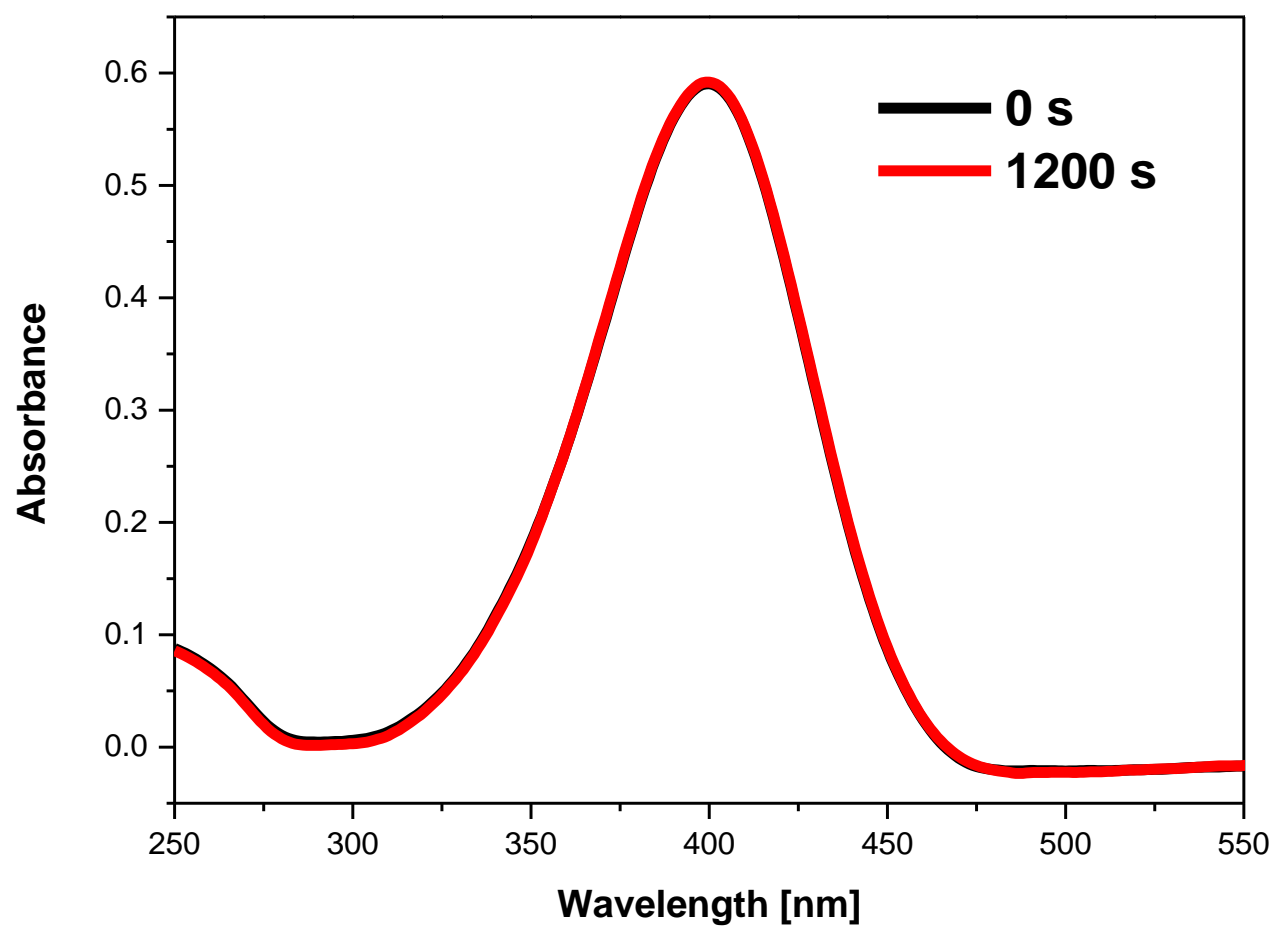

Figure S6. UV-Vis absorption spectra after pure pored hollow PS microparticles (i.e. not covered with Ag nanocrystals) were added to the solution containing $\mathrm{NaBH}_{4}$ and 4-NP. 


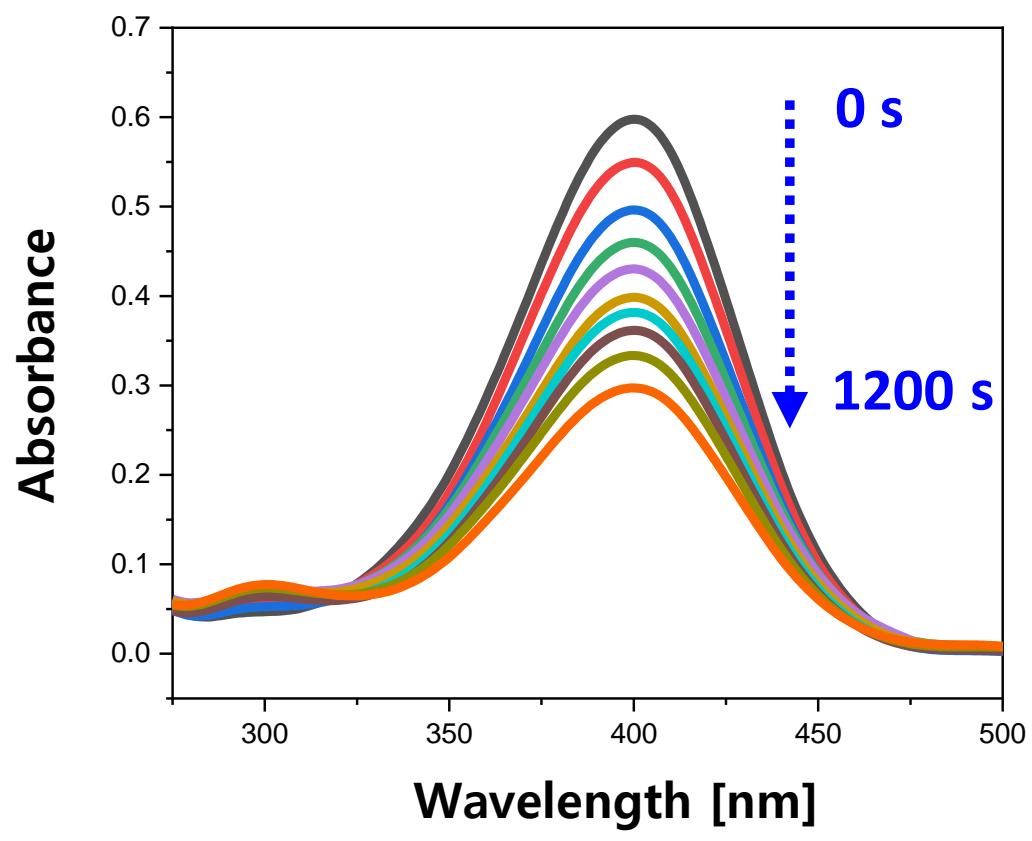

Figure S7. Time-dependent UV-Vis absorption spectra for the catalytic reduction of 4-NP to 4AP in aqueous medium after Ag nanocrystals/hollow and dimpled PS hybrid microparticles were added to the solution. 